\title{
Lattice Results for Vector Meson Couplings and Parton Distribution Amplitudes
}

\section{UKQCD and RBC Collaborations}

\section{M.A. Donnellan, J. Flynn, A. Jüttner, C.T. Sachrajda*}

UKQCD Collaboration School of Physics and Astronomy, University of Southampton, Southampton SO17 1BJ UK

E-mail: mad2@phys.soton.ac.uk, jflynn@phys.soton.ac.uk, juettner@phys.soton.ac.uk, cts@phys.soton.ac.uk

\section{Antonio, P.A. Boyle, C. Maynard, B. Pendleton, R.Tweedie}

School of Physics, The University of Edinburgh, Edinburgh, EH9 3JZ UK

E-mail: s0459477@sms.ed.ac.uk, paboyle@ph.ed.ac.uk

c.maynard@ed.ac.uk, bjp@ph.ed.ac.uk, rjt@ph.ed.ac.uk

\begin{abstract}
We present results for the couplings of light vector mesons to vector and tensor currents and on the low moments of the light-cone distribution amplitudes of the pion and kaon. The calculations are performed on the RBC and UKQCD collaborations' ensembles generated with the Iwasaki gauge action and with $2+1$ flavours of domain wall fermions. The (preliminary) results for the ratios of the couplings of the vector meson to the vector and tensor currents ( $f_{V}$ and $f_{V}^{T}$ respectively) in the $\overline{\mathrm{MS}}$ scheme at $2 \mathrm{GeV}$ are: $f_{\rho}^{T} / f_{\rho}=0.681(20) ; f_{K^{*}}^{T} / f_{K^{*}}=0.712(11)$ and $f_{\phi}^{T} / f_{\phi}=0.751(9)$. For the first moment of the kaon's distribution amplitude we find (in the same scheme and at the same scale) $\langle\xi\rangle_{K}=0.029(2)$ and for the second moment $\left\langle\xi^{2}\right\rangle_{\pi}=0.28(3)$ and $\left\langle\xi^{2}\right\rangle_{K}=0.27(2)$.
\end{abstract}

The XXV International Symposium on Lattice Field Theory

July 30-4 August 2007

Regensburg, Germany

\footnotetext{
* Speaker.
} 


\section{Introduction}

In this talk I briefly review the status of calculations by the RBC and UKQCD collaborations of the couplings of light vector mesons to vector and tensor currents and of the low moments of the light-cone distribution amplitudes of the pion and kaon. (A detailed description will be presented in forthcoming publications.) We use the collaborations' two principal ensembles, generated with the Iwasaki gauge action and domain wall fermions with an inverse lattice spacing of about $1.73 \mathrm{GeV}$. The two lattices have $24^{3} \times 64 \times 16$ points (corresponding to a physical volume of $L \simeq 2.7 \mathrm{fm}$ ) and $16^{3} \times 32 \times 16$ points (physical volume, $L \simeq 1.8 \mathrm{fm}$ ). The properties of the $16^{3}$ (spatial) lattice have been presented in detail in ref. [1] and those of the $24^{3}$ one have been summarised in the plenary talk by Peter Boyle at this conference [2] and the corresponding paper is being prepared for publication.

On the $24^{3}$ lattice, measurements have been made using 4 values of the light-quark mass whose bare values (and the corresponding pion masses) are as follows:

$$
\begin{array}{ll}
m a=0.03\left(m_{\pi} \simeq 670 \mathrm{MeV}\right) ; & m a=0.02\left(m_{\pi} \simeq 555 \mathrm{MeV}\right) ; \\
m a=0.01\left(m_{\pi} \simeq 415 \mathrm{MeV}\right) ; & m a=0.005\left(m_{\pi} \simeq 330 \mathrm{MeV}\right),
\end{array}
$$

where $a$ is the lattice spacing. On the $16^{3}$ lattice results were obtained with $m a=0.03,0.02$ and 0.01 . For the strange quark we take $m_{s} a=0.04$ and the residual mass is $m_{\text {res }} a=0.00325(2)$.

On the $16^{3}$ lattice it was found that $a^{-1}=1.62(4) \mathrm{GeV}$ and $m_{s} a=0.04$ [1]. With the new data on the $24^{3}$ lattice it is possible to reach lower masses and perform a more detailed investigation of the chiral behaviour [2,3]. The conclusion is that $a^{-1}=1.73(3) \mathrm{GeV}$ and $m_{s} a \simeq 0.0344(16)$ and below I will discuss the corresponding corrections to the results obtained directly with $m_{s} a=0.04$.

\section{Couplings of Vector Mesons to Vector and Tensor Currents}

The couplings of the vector meson to the vector and tensor currents ( $f_{V}$ and $f_{V}^{T}$ respectively) are defined through the matrix elements:

$$
\begin{aligned}
\left\langle 0\left|\bar{q}_{2}(0) \gamma^{\mu} q_{1}(0)\right| V(p ; \lambda)\right\rangle & =f_{V} m_{V} \varepsilon_{\lambda}^{\mu} \\
\left\langle 0\left|\bar{q}_{2}(0) \sigma^{\mu v} q_{1}(0)\right| V(p ; \lambda)\right\rangle & =i f_{V}^{T}(\mu)\left(\varepsilon_{\lambda}^{\mu} p^{v}-\varepsilon_{\lambda}^{v} p^{\mu}\right),
\end{aligned}
$$

where $p$ and $\lambda$ are the momentum and polarization state of the vector meson $V(p ; \lambda)$ and $\varepsilon_{\lambda}$ is the corresponding polarization vector. The tensor bilinear operator $\bar{q}_{2} \sigma^{\mu v} q_{1}$ (and hence $f_{V}^{T}(\mu)$ ) depends on the renormalization scheme and scale $\mu$. The final results will be quoted in the $\overline{\mathrm{MS}}$ scheme at $\mu=2 \mathrm{GeV}$.

\subsection{Experimental Determination of $f_{V}$}

The decay constants $f_{V}$ can be determined experimentally. For the charged $\rho$ and $K^{*}$ mesons, one can use $\tau$ decays to deduce $f_{\rho}$ and $f_{K^{*}}$ as illustrated by the following diagram:

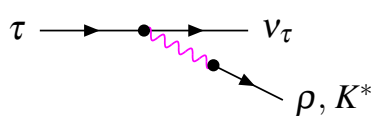


From the measured branching ratios one obtains the following values for the decay constants [4]:

$$
\begin{aligned}
\operatorname{Br}\left(\tau^{-} \rightarrow \rho^{-} v_{\tau}\right)=(25.0 \pm 0.3) \% & \Rightarrow f_{\rho^{-}} \simeq 208 \mathrm{MeV} \\
\operatorname{Br}\left(\tau^{-} \rightarrow K^{*-} v_{\tau}\right)=(1.29 \pm 0.03) \% & \Rightarrow f_{K^{*-}} \simeq 217 \mathrm{MeV}
\end{aligned}
$$

One can also determine $f_{\rho^{0}}$ from the width of the decay of the $\rho^{0}$ into $e^{+} e^{-}$which gives $f_{\rho^{0}}=$ $216(5) \mathrm{MeV}$. Similarly from the width of the decay $\phi \rightarrow e^{+} e^{-}$one deduces $f_{\phi} \simeq 233 \mathrm{MeV}$.

The couplings $f_{V}^{T}$ are not known from experiment but are used as inputs in sum-rule calculations and other phenomenological applications. I will present our results for $f_{V}^{T} / f_{V}$, which can then be combined with the experimental values of $f_{V}$ to obtain $f_{V}^{T}$. For the $\phi$ we neglect the Zweig suppressed disconnected contribution.

\subsection{Lattice Calculation of $f_{V}^{T} / f_{V}$}

In order to determine $f_{V}^{T} / f_{V}$ it is sufficient to calculate the following zero-momentum correlation functions:

$$
\begin{aligned}
& C_{V^{s_{1}} V^{s_{2}}}(t) \equiv \sum_{\vec{x}, i}\left\langle 0\left|V_{i}^{s_{1}}(t, \vec{x}) V_{i}^{s_{2}}(0)\right| 0\right\rangle=3 f_{V}^{s_{1}} f_{V}^{s_{2}} m_{V} e^{-m_{V} T / 2} \cosh \left(m_{V}(T / 2-t)\right) \\
& C_{V^{s_{1}} T^{s_{2}}}(t) \equiv \sum_{\vec{x}, i}\left\langle 0\left|V_{i}^{s_{1}}(t, \vec{x}) T_{4 i}^{s_{2}}(0)\right| 0\right\rangle=3 f_{V}^{s_{1}} f_{V}^{T s_{2}} m_{V} e^{-m_{V} T / 2} \sinh \left(m_{V}(T / 2-t)\right),
\end{aligned}
$$

where $V_{\mu}$ and $T_{\mu v}$ represent the vector and tensor current and $s_{1}$ and $s_{2}$ label the smearing at the sink and source respectively. $i$ is a spatial index $(i=1,2,3)$. From the ratio

$$
R(t)=\frac{C_{V^{s_{1}} T^{L}}}{C_{V^{s_{1}} V^{L}}}=\frac{f_{V}^{T}}{f_{V}} \tanh \left(m_{V}(T / 2-t)\right)
$$

we readily obtain the ratio of (bare) couplings. In practice we also calculate the correlation functions $C_{T^{s_{1} V^{s_{2}}}}(t)$ and include them in the analysis. For the smearing we use a gauge invariant Gaussian and hydrogen-like gauge-fixed wave function.

\subsection{Results}

In table 1 we present the bare values of $f_{V}^{T} / f_{V}$ corresponding to both the lattice volumes. It can be seen that the measured results are obtained with excellent precision. For the two heaviest quark masses the results from the two volumes are fully compatible; for $m a=0.01$ perhaps there is a hint of a small finite volume effect and we do not include the results from the $16^{3}$ lattice at this mass in the final analysis.

From fig. 1 it can be seen that the dependence of the bare $f_{V}^{T} / f_{V}$ on the masses of the light quarks is very mild. For the ratio of bare couplings in the chiral limit we obtain:

$$
\frac{f_{\rho}^{T}}{f_{\rho}}=0.624(13)_{(-21)}^{(+0)} ; \quad \frac{f_{K^{*}}^{T}}{f_{K^{*}}}=0.6505(56)_{(-115)}^{(+0)} ; \quad \frac{f_{\phi}^{T}}{f_{\phi}}=0.6824(31)_{(-0)}^{(+35)},
$$

where the central value corresponds to the linear extrapolation and the second error is the difference between the results from the linear and quadratic extrapolations.

The bare results in eq. (2.8) were obtained with the "notional" strange quark mass of $m_{s} a=$ 0.04 rather than the updated value of 0.0344 . The values of the ratios in eq. (2.8) are very similar 


\begin{tabular}{lccccc}
\hline \hline & Volume & $m a=0.03$ & $m a=0.02$ & $m a=0.01$ & $m a=0.005$ \\
\hline$f_{\rho}^{T} / f_{\rho}$ & $24^{3}$ & $0.6813(47)$ & $0.6699(76)$ & $0.648(12)$ & $0.624(23)$ \\
$f_{\rho}^{T} / f_{\rho}$ & $16^{3}$ & $0.6885(63)$ & $0.6660(69)$ & $0.6236(80)$ & - \\
\hline$f_{K^{*}}^{T} / f_{K^{*}}$ & $24^{3}$ & $0.6872(70)$ & $0.6826(61)$ & $0.6673(43)$ & $0.6556(60)$ \\
$f_{K^{*}}^{T} / f_{K^{*}}$ & $16^{3}$ & $0.6879(48)$ & $0.6774(45)$ & $0.6516(46)$ & - \\
\hline$f_{\phi}^{T} / f_{\phi}$ & $24^{3}$ & $0.6929(49)$ & $0.6942(40)$ & $0.6876(24)$ & $0.6868(28)$ \\
$f_{\phi}^{T} / f_{\phi}$ & $16^{3}$ & $0.7023(47)$ & $0.6907(31)$ & $0.6822(33)$ & - \\
\hline \hline
\end{tabular}

Table 1: Results for the bare ratios of couplings $f_{V}^{T} / f_{V}$ obtained on both the lattice volumes.
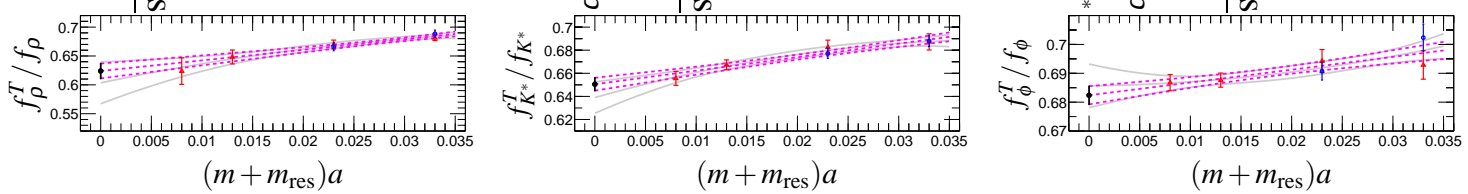

Figure 1: Chiral extrapolations for $f_{\rho}^{T} / f_{\rho}, f_{K^{*}}^{T} / f_{K^{*}}$ and $f_{\phi}^{T} / f_{\phi}$ respectively. The broken red lines represent a linear fit to the mass behaviour and the solid grey lines a quadratic fit.

for the $\rho, K^{*}$ and $\phi$ mesons and we correct for the change in $m_{s} a$ by linear extrapolation in the valence quark mass ( $a m_{s}^{\text {sea }}$ is fixed at 0.04 ). Thus, for example, for the $K^{*}$ meson we write:

$$
\frac{f_{K^{*}}^{T}}{f_{K^{*}}}\left(m_{s} a=0.0344\right)=\frac{f_{K^{*}}^{T}}{f_{K^{*}}}\left(m_{s} a=0.04\right)+\frac{\Delta}{\left(0.04+a m_{\mathrm{res}}\right)}(0.0344-0.04),
$$

where $\Delta=f_{K^{*}}^{T} / f_{K^{*}}\left(m_{s} a=0.04\right)-f_{\rho}^{T} / f_{\rho}$. The corrected bare values are then

$$
\frac{f_{\rho}^{T}}{f_{\rho}}=0.624(13)_{(-21)}^{(+0)} ; \quad \frac{f_{K^{*}}^{T}}{f_{K^{*}}}=0.6470(56)_{(-115)}^{(+0)} ; \quad \frac{f_{\phi}^{T}}{f_{\phi}}=0.6748(31)_{(-0)}^{(+35)}(30),
$$

where we have added the third error on $f_{\phi}^{T} / f_{\phi}$ to reflect the uncertainty on the strange quark mass and to allow for non-linearities in the behaviour (for the $K^{*}$ the corresponding error is negligible).

We determine the renormalization constants non-perturbatively using the Rome-Southampton method and run the results to $2 \mathrm{GeV}[5]$ :

$$
\frac{f_{V}^{T}(2 \mathrm{GeV})}{f_{V}}=\frac{Z_{T}(2 \mathrm{GeV} a)}{Z_{V}} \frac{f_{V}^{T \text { bare }}(a)}{f_{V}^{\text {bare }}}=1.11(1) \frac{f_{V}^{T \text { bare }}(a)}{f_{V}^{\text {bare }}} .
$$

In the $\overline{\mathrm{MS}}$ scheme with $\mu=2 \mathrm{GeV}$ we finally obtain:

$$
\frac{f_{\rho}^{T}}{f_{\rho}}=0.681(20) ; \quad \frac{f_{K^{*}}^{T}}{f_{K^{*}}}=0.712(11) ; \quad \frac{f_{\phi}^{T}}{f_{\phi}}=0.751(9) .
$$

These results can be compared with previous quenched lattice results: 


\begin{tabular}{c|c|c|c} 
Reference & $\frac{f_{\rho}^{T}(2 \mathrm{GeV})}{f_{\rho}}$ & $\frac{f_{K^{*}}^{T}(2 \mathrm{GeV})}{f_{K^{*}}}$ & $\frac{f_{\phi}^{T}(2 \mathrm{GeV})}{f_{\phi}}$ \\
\hline Becirevic et al. [4] & $0.720 \pm 0.024_{-0.000}^{+0.016}$ & $0.739 \pm 0.017_{-0.000}^{+0.003}$ & $0.759 \pm 0.009 \pm 0.000$ \\
Braun et al. [6] & $0.742 \pm 0.014$ & - & $0.780 \pm 0.008$ \\
\hline
\end{tabular}

The QCDSF/UKQCD also presented the result $f_{\rho}^{T}=168(3) \mathrm{MeV}$ at Lattice 2005, using an $N_{f}=2 O(a)$ improved clover action with a range of lattice spacings $(0.07<a<0.11 \mathrm{fm})$ [7]. Combining our result for the ratio from eq. (2.12) together with the experimental value for $f_{\rho}$ we obtain a smaller value $f_{\rho}^{T}=140(5) \mathrm{MeV}$.

\section{Pion and Kaon Distribution Amplitudes}

The leading-twist distribution amplitude of the pion, $\phi_{\pi}$, is defined by:

$$
\left.\left\langle\pi^{+}(q)\left|\bar{u}_{\alpha}(z) \mathscr{P}(z,-z) d_{\beta}(-z)\right| 0\right\rangle\right|_{z^{2}=0} \equiv \frac{i f_{\pi}}{4}\left(\not q \gamma_{5}\right)_{\beta \alpha} \int_{0}^{1} d u e^{i(2 u-1) q \cdot z} \phi_{\pi}(u, \mu),
$$

where $\mathscr{P}$ is the path-ordered exponential. For the kaon there is a similar definition with the obvious change of quark flavours. The (universal) distribution amplitudes contain the non-perturbative QCD effects in hard exclusive processes.

Performing a series expansion to first order in $z$ :

$$
\left\langle\pi^{+}(q)\left|\bar{u}(0) \gamma_{5} \gamma_{\{\rho} \stackrel{\leftrightarrow}{D}_{\mu\}} d(0)\right| 0\right\rangle=f_{\pi}\left(i q_{\mu}\right)\left(i q_{\rho}\right) \int_{0}^{1} d u(2 u-1) \phi_{\pi}(u, \mu) \equiv f_{\pi}\left(i q_{\mu}\right)\left(i q_{\rho}\right)\langle\xi\rangle_{\pi}
$$

$\langle\xi\rangle_{\pi}=0$ by isospin symmetry; we calculate $\langle\xi\rangle_{K}$.

Performing the series expansion to next order we have:

$$
\left\langle\pi^{+}(q)\left|\bar{u} \gamma_{5} \gamma_{\{\rho} \stackrel{\leftrightarrow}{D}_{\mu} \stackrel{\leftrightarrow}{D}_{v\}} d\right| 0\right\rangle=f_{\pi}\left(i q_{\rho}\right)\left(i q_{\mu}\right)\left(i q_{v}\right) \int_{0}^{1} d u(2 u-1)^{2} \phi_{\pi}(u, \mu)
$$

We define $\left\langle\xi^{2}\right\rangle_{\pi} \equiv \int_{0}^{1} d u(2 u-1)^{2} \phi_{\pi}(u, \mu)$ and similarly for the kaon.

The lattice determination of $\langle\xi\rangle_{K}^{\text {bare }}$ is very straightforward. We compute the ratio of two point correlation functions:

$$
\frac{\sum_{\vec{x}} e^{i \vec{p} \cdot \vec{x}}\left\langle 0\left|O_{\{\rho \mu\}}(t, \vec{x}) P^{\dagger}(0)\right| 0\right\rangle}{\sum_{\vec{x}} e^{i \vec{p} \cdot \vec{x}}\left\langle 0\left|A_{v}(t, \vec{x}) P^{\dagger}(0)\right| 0\right\rangle} \quad \underset{t \text { large }}{\longrightarrow} i \frac{p_{\rho} p_{\mu}}{p_{v}}\langle\xi\rangle_{K}^{\text {bare }}
$$

where $P$ and $A_{v}$ are the pseudoscalar and axial bilinears and $O_{\{\rho \mu\}}=\bar{u} \gamma_{5} \gamma_{\{\rho} \stackrel{\leftrightarrow}{D}_{\mu\}} s$. The most appropriate choice of indices is $O_{\{4 i\}}$ and $v=4$. The second moment is evaluated from a similar ratio with $O_{\{4 i j\}}=\bar{u} \gamma_{5} \gamma_{\{4} \stackrel{\leftrightarrow}{D_{i}} \stackrel{\leftrightarrow}{D}_{j\}} s$ and $i \neq j$. The transformation properties of the operators under the lattice symmetries have been studied in detail $[8,9]$.

\subsection{Results for $\langle\xi\rangle_{K}$}

In fig. 2 we plot our bare results as a function of the mass of the light quark with $m_{s} a=0.04$. The three points without a black border were obtained on the $16^{3}$ lattice and the results were 


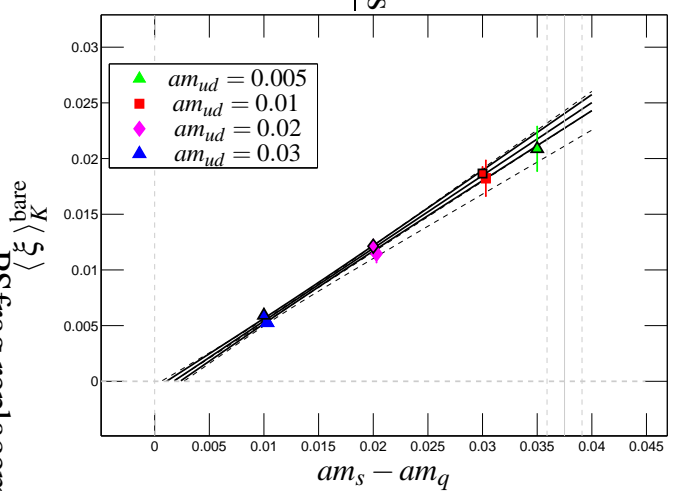

Figure 2: Bare values of $\langle\xi\rangle_{K}$ vs the quark mass. The physical region $m_{s} a-m_{q} a=0.0375(16)$ is marked.
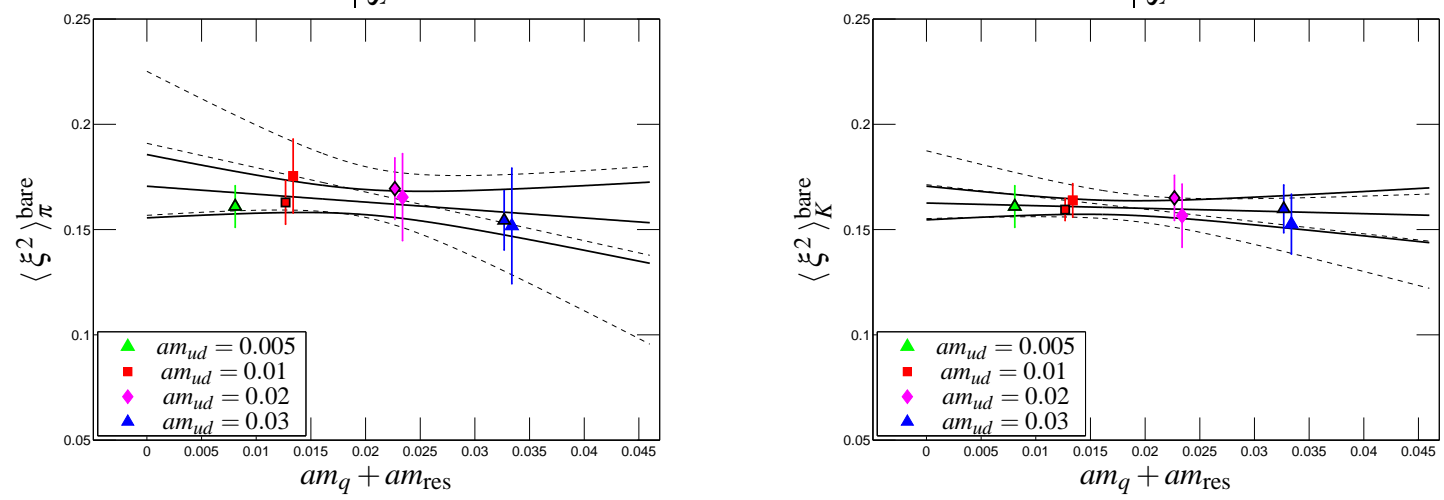

Figure 3: Bare values of $\left\langle\xi^{2}\right\rangle_{\pi}$ and $\left\langle\xi^{2}\right\rangle_{K}$ as a function of the quark mass.

presented in ref. [10]. The remaining four points (including the point at $m a=0.005$ ) were obtained from the $24^{3}$ lattice and there is no evidence of significant finite volume effects. $\langle\xi\rangle_{K}$ is clearly non-zero away from the $S U(3)$ limit and the results are compatible with the prediction from lowest order chiral perturbation theory that $\langle\xi\rangle_{K}$ is proportional to $m_{s}-m_{q}$ without logarithms [11]. The linearly extrapolated value in the flavour symmetry limit $\left(m a=m_{s} a=0.04\right)$ is approximately 0 . The bare result in the physical limit $\left(\left(m_{s}-m_{q}\right) a=0.0375(16)\right.$, see fig. 2) obtained with a linear fit is $\langle\xi\rangle_{K}^{\text {bare }}=0.0234(12)$. Multiplying this result by the renormalization factor (which, so far we have only calculated perturbatively) we finally obtain:

$$
\langle\xi\rangle_{K}^{\overline{\mathrm{MS}}}(2 \mathrm{GeV})=0.029(2) .
$$

\subsection{Results for $\left\langle\xi^{2}\right\rangle$}

Figure 3 contains our results for $\left\langle\xi^{2}\right\rangle_{\pi}^{\text {bare }}$ and $\left\langle\xi^{2}\right\rangle_{K}^{\text {bare }}$ from which we deduce the values in the chiral limit: $\left\langle\xi^{2}\right\rangle_{\pi}^{\text {bare }}=0.171(15)$ and $\left\langle\xi^{2}\right\rangle_{K}^{\text {bare }}=0.163(8)$. In order to obtain the results in the $\overline{\mathrm{MS}}$ scheme, we need to perform the renormalization, which up to now we have done perturbatively. The operator $O_{D D}=\bar{\psi} \gamma_{\{\mu} \gamma_{5} \stackrel{\leftrightarrow}{D}_{v} \stackrel{\leftrightarrow}{D}_{\kappa\} \psi}$ (with $\mu, v$ and $\kappa$ all distinct) mixes with $O_{\partial \partial}=\partial_{\{v} \partial_{\kappa} \bar{\psi} \gamma_{\mu\}} \gamma_{5} \psi$ Dividing by the matrix element of the axial current we find

$$
\left\langle\xi^{2}\right\rangle^{\overline{\mathrm{MS}}}(a)=1.52(7)\left\langle\xi^{2}\right\rangle^{\text {bare }}+0.022(7) .
$$

Inserting the measured values of $\left\langle\xi^{2}\right\rangle^{\text {bare }}$ and (two-loop) running the result to $2 \mathrm{GeV}$ we obtain

$$
\left\langle\xi^{2}\right\rangle_{\pi}^{\overline{\mathrm{MS}}}(2 \mathrm{GeV})=0.278 \pm 0.026 \text { and }\left\langle\xi^{2}\right\rangle_{K}^{\overline{\mathrm{MS}}}(2 \mathrm{GeV})=0.267 \pm 0.018
$$


Since $\left\langle\xi^{2}\right\rangle_{\pi}=\left\langle\xi^{2}\right\rangle_{K}$ to excellent precision, we do not make any interpolation to $m_{s} a=0.0344$.

\subsection{Final Comments}

We have shown that lattice calculations of $\langle\xi\rangle_{K}$, and $\left\langle\xi^{2}\right\rangle_{\pi, K}$ can be performed with an excellent precision. Our (preliminary) results are:

$$
\langle\xi\rangle_{K}^{\overline{\mathrm{MS}}}(2 \mathrm{GeV})=0.029(2), \quad\left\langle\xi^{2}\right\rangle_{\pi}^{\overline{\mathrm{MS}}}(2 \mathrm{GeV})=0.28(3), \quad\left\langle\xi^{2}\right\rangle_{K}^{\overline{\mathrm{MS}}}(2 \mathrm{GeV})=0.27(2) .
$$

Important improvements will be to perform the non-perturbative renormalization and to repeat the calculation at a finer lattice spacing (we estimate a further 5\% error due to discretization effects). Our results are in agreement with those of an $N_{f}=2$ study using Improved Wilson fermions [12]

$$
\langle\xi\rangle_{K}^{\overline{\mathrm{MS}}}(2 \mathrm{GeV})=0.0272(5), \quad\left\langle\xi^{2}\right\rangle_{\pi}^{\overline{\mathrm{MS}}}(2 \mathrm{GeV})=0.269(39), \quad\left\langle\xi^{2}\right\rangle_{K}^{\overline{\mathrm{MS}}}(2 \mathrm{GeV})=0.260(6) .
$$

The analysis of moments of the distribution amplitudes of vector mesons is in progress.

\section{References}

[1] C. Allton et al. [RBC \& UKQCD Collaborations], 2+1 flavor domain wall QCD on a $(2-f m)^{3}$ lattice: Light meson spectroscopy with $L_{s}=16$, Phys. Rev. D 76 (2007) 014504 [arXiv: hep-lat/0701013].

[2] P.A. Boyle, 2+1 flavour domain wall fermion simulations by the RBC and UKQCD collaborations, these proceedings.

[3] M. Lin and E.E. Scholz, Chiral limit and light quark masses in $2+1$ flavor domain wall QCD, these proceedings.

[4] D. Becirevic, V. Lubicz, F. Mescia and C. Tarantino, Coupling of the light vector meson to the vector and to the tensor current, JHEP 0305 (2003) 007 [ arXiv: hep-lat/0301020] .

[5] Y. Aoki et al. (RBC \& UKQCD Collaborations), paper in preparation.

[6] V. M. Braun, T. Burch, C. Gattringer, M. Gockeler, G. Lacagnina, S. Schaefer and A. Schafer, A lattice calculation of vector meson couplings to the vector and tensor currents using chirally improved fermions, Phys. Rev. D 68 (2003) 054501 [arXiv:hep-lat/0306006].

[7] M. Gockeler et al., Meson decay constants from $N(f)=2$ clover fermions, PoS LAT2005 (2006) 063 [arXiv:hep-lat/0509196].

[8] G. Martinelli and C. T. Sachrajda, A Lattice Calculation of the Pion's Form-Factor and Structure Function, Nucl. Phys. B 306 (1988) 865.

[9] M. Gockeler, R. Horsley, E. M. Ilgenfritz, H. Perlt, P. Rakow, G. Schierholz and A. Schiller, Lattice Operators for Moments of the Structure Functions and their Transformation under the Hypercubic Group, Phys. Rev. D 54 (1996) 5705 [arXiv:hep-lat/9602029].

[10] P. A. Boyle, M. A. Donnellan, J. M. Flynn, A. Juttner, J. Noaki, C. T. Sachrajda and R. J. Tweedie, [UKQCD Collaboration], A lattice computation of the first moment of the kaon's distribution amplitude, Phys. Lett. B 641 (2006) 67 [arXiv:hep-lat/0607018].

[11] J. W. Chen and I. W. Stewart, Model independent results for $S U(3)$ violation in light-cone distribution functions, Phys. Rev. Lett. 92 (2004) 202001 [arXiv:hep-ph/0311285].

[12] V. M. Braun et al., Moments of pseudoscalar meson distribution amplitudes from the lattice, Phys. Rev. D 74 (2006) 074501 [arXiv:hep-lat/0606012]. 